\title{
Objective-Oriented Utility-Based Association Mining
}

\author{
Yi-Dong Shen \\ Laboratory of Computer Science \\ Institute of Software, Chinese Academy of Sciences \\ Beijing 100080, P. R. China \\ ydshen@ios.ac.cn
}

\author{
Qiang Yang and Zhong Zhang \\ School of Computing Science \\ Simon Fraser University \\ Burnaby, BC, Canada V5A 1S6 \\ \{qyang, zzhang\}@cs.sfu.ca
}

\begin{abstract}
The necessity to develop methods for discovering association patterns to increase business utility of an enterprise has long been recognized in data mining community. This requires modeling specific association patterns that are both statistically (based on support and confidence) and semantically (based on objective utility) relating to a given objective that a user wants to achieve or is interested in. However, we notice that no such a general model has been reported in the literature. Traditional association mining focuses on deriving correlations among a set of items and their association rules like diaper $\rightarrow$ beer only tell us that a pattern like \{diaper $\}$ is statistically related to an item like beer. In this paper, we present a new approach, called Objective-Oriented utility-based Association (OOA) mining, to modeling such association patterns that are explicitly relating to a user's objective and its utility. Due to its focus on a user's objective and the use of objective utility as key semantic information to measure the usefulness of association patterns, OOA mining differs significantly from existing approaches such as the existing constraint-based association mining. We formally define OOA mining and develop an algorithm for mining OOA rules. The algorithm is an enhancement to Apriori with specific mechanisms for handling objective utility. We prove that the utility constraint is neither monotone nor anti-monotone nor succinct nor convertible and present a novel pruning strategy based on the utility constraint to improve the efficiency of OOA mining.
\end{abstract}

\section{Introduction}

Association mining is an important problem in data mining. Briefly, given a historical dataset of an application, we derive frequent patterns and association rules from the dataset by using some thresholds, such as a minimum support and a minimum confidence. Since Agrawal's pioneer work [1], a lot of research has been conducted on asso- ciation mining. Major achievements include approaches to improving the efficiency of computing the frequent patterns from large datasets [1, 4], approaches to applying constraints to find more interesting patterns [2, 10, 14], and approaches to eliminating irrelevant association rules by making use of some interestingness measures [8, 13].

Observe that most existing approaches to association mining are itemset-correlation-oriented in the sense that they aim to find out how a set of items are statistically correlated by mining association rules of the form

$$
I_{1}, \ldots, I_{m} \rightarrow I_{m+1}(s \%, c \%)
$$

where $s \%$, the support of the rule, is the probability of all items $I_{1}, \ldots, I_{m+1}$ occurring together, and $c \%$, the confidence of the rule, is the conditional probability of $I_{m+1}$ given the itemset $\left\{I_{1}, \ldots, I_{m}\right\}$. Both $s \%$ and $c \%$ are obtained simply by counting the frequency of the respective itemsets in a given dataset, and are greater than or equal to the user-specified minimum support and minimum confidence, respectively.

Although finding correlations of itemsets like diaper $\rightarrow$ beer is very important, in many situations people may be more interested in finding out how a set of items support a specific objective $O b j$ that they want to achieve by discovering association rules of the form

$$
I_{1}, \ldots, I_{m} \rightarrow \operatorname{Obj}(s \%, c \%, u)
$$

where (1) $s \%$ (the support of the rule) is the probability that all items $I_{1}, \ldots, I_{m}$ together with $O b j$ hold, (2) $c \%$ (the confidence of the rule) is the conditional probability of $O b j$ given the itemset $\left\{I_{1}, \ldots, I_{m}\right\}$, and (3) $u$ is the utility of the rule, showing to what degree the pattern $\left\{I_{1}, \ldots, I_{m}\right\}$ semantically supports $O b j$. Due to its focus on an objective and the use of objective utility as key semantic information to measure the usefulness of association patterns, we refer to this new type of association mining as ObjectiveOriented utility-based Association (OOA) mining, as opposed to traditional Itemset-Correlation-Oriented Association (ICOA) mining. 
OOA mining derives patterns that both statistically and semantically support a given objective $O b j$. Informally, $I=\left\{I_{1}, \ldots, I_{m}\right\}$ is said to statistically support $O b j$ if the support $s \%$ and confidence $c \%$ of the rule (2) are not below a user-specified minimum support $m s \%$ and a userspecified minimum confidence $m c \%$, respectively. And $I$ is said to semantically support $O b j$ if the utility $u$ of the rule (2) is not below a user-specified minimum utility $m u$. As a result, all patterns derived in OOA mining must be interesting to an enterprise since when employed, they would increase the (expected) utility of the enterprise above the user-specified minimum level $(u \geq m u)$. Therefore, OOA mining has wide applications in many areas where people are looking for objective-centered statistical solutions to achieve their goals. For a typical example, in business situations a manager may use OOA mining to discover the best business strategies by specifying his/her objective as "high profit and low risk of loss." Another example is in medical field. A doctor may use OOA mining to find the best treatments for a disease by specifying an objective "high effectiveness and low side-effects."

The term utility is commonly used to mean "the quality of being useful" and utilities are widely used in decision making processes to express user's preferences over decision objects towards decision objectives [5, 12]. In decision theory, we have the well-known equation "Decision = probability + utility," which says that a decision object is chosen based on its probability and utility. Since association mining can be viewed as a special decision problem where decision objects are patterns, we may well have, correspondingly, an equation "Interestingness (of a pattern) = probability + utility." This equation further justifies the necessity and significance of enhancing traditional probability (support and confidence) based association mining with objective related utilities.

Since utilities are subjective, they can be acquired from domain experts/users. We would point out, however, that this does not mean we need to acquire a utility for each single item in a dataset. As we will see in Section 3, it suffices to obtain utilities only for those items in a dataset which are directly related to the given objective. The population of such objective items would be quite small in practical applications.

In this paper, we systematically study OOA mining. In Section 3, we formally define the concepts of objective, support, confidence, and utility under the frame of OOA mining. In particular, we will present a formulation of an objective and define utilities based on the formulation. In Section 4 , we develop an algorithm for mining OOA frequent patterns and rules. The algorithm is based on Apriori, with an enhancement that handles objective utility. Traditional association mining is NP-hard [16], but OOA mining does not seem to be easier. To improve the efficiency of OOA min- ing, we will present a novel strategy for pruning itemsets based on the support and utility constraints. In Section 5, we present some experimental results.

\section{Related Work}

The necessity to develop methods for finding specific patterns which can be used to increase business utility has long been recognized by several researchers $[6,9,13]$. To the best of our knowledge, however, no work on association mining has been reported in the literature which formally models such patterns that are explicitly relating to a user's objective and its utility. In this paper, we develop such a model. An OOA rule $I_{1}, \ldots, I_{m} \rightarrow O b j$ not only shows that the pattern $\left\{I_{1}, \ldots, I_{m}\right\}$ statistically supports the user's objective $O b j$, but also suggests that when being applied to the underlying enterprise it would increase the expected utility above a user-specified minimum level.

Our work is related to but different from existing constrained association mining. Existing constrained association mining, typically represented by the work of Bayardo, Agrawal, and Gounopolos [2], Han, Lakshmanan, Ng, Pang and Pei [10,11], and Srikant, Agrawal and Vu [14], takes the form $\{(S \rightarrow T) \mid C\}$ where $S$ and $T$ are sets of items and $C$ is a set of constraints on the selection of $S$ and $T$. When $T$ is not empty, such kind of association mining belongs to ICOA mining because no matter what constraints $C$ is, it always derives asociation rules of the form $I_{1}, \ldots, I_{m} \rightarrow J_{1}, \ldots, J_{n}$ where both itemsets $\left\{I_{1}, \ldots, I_{m}\right\}$ and $\left\{J_{1}, \ldots, J_{n}\right\}$ satisfy $C$. Certainly, OOA mining can use constraints, too. Constrained OOA mining takes the form $\left\{(S \rightarrow O b j) \mid C_{o b j}\right\}$ where $C_{o b j}$ is a set of constraints on the selection of $S$ in terms of the objective $O b j$. Constrained OOA mining always derives OOA rules.

Another significant difference between existing constrained association mining and OOA mining is that most exisitng work focuses on SQL-style constraints including item selection, pattern length, set relations $(\subseteq, \supseteq$, etc.), $\max (S) \theta v, \min (S) \theta v, \operatorname{sum}(S) \theta v, \operatorname{count}(S) \theta v$ and $\operatorname{avg}(S) \theta v$, where $S$ is an itemset, $v$ is a real number, and $\theta$ is $\leq$ or $\geq$ (see [11] for a summary of types of constraints discussed in the literature). These constraints fall into one of the following four well-defined categories: monotone, antimonotone, succinct or convertible. In OOA mining, however, we introduce objective utility as a key constraint. On the one hand, an (arbitrary) objective and its utility are difficult, if not impossible, to be formulated using SQL-style constraints. On the other hand, the utility constraint is neither monotone nor anti-monotone nor succinct nor convertible (see Section 4.2 for the proof). Therefore, no existing constrained association mining methods are applicable to it. In this work we push the utility constraint deep into OOApriori (a variant of Apriori) to prune candicate patterns 
in order to efficiently derive all OOA rules.

We would point out that although business objectives, such as "high profit and low risk of loss," can be viewed as constraints, such constraints seem to be at a meta-level w.r.t. the above mentioned SQL-style constraints. Therefore, specific mechanisms are required to represent and handle them. The proposed OOA mining may then be the first such mechanism.

Most recently, Wang, Zhou and Han [15] and Lin, Yao and Louie [7] suggested adding values to association rules. The former takes into account the price and quantity of supermaket sales during association mining, while the latter tries to attach a value to every item in a dataset and use the added values to rank association rules. There are three major differences between their approaches and ours. First, we do general objective centered mining by explicitly declaring a user's objective and formulating it in a simple, uniform way (see Section 3). As a result, utilities are assigned only to those items which directly contribute to the objective. Second, we handle both positive and negative utilities, whereas they only consider positive values. Negative utility represents punishment/loss, and it is with negative values that our utility constraints become neither monotone nor anti-monotone nor succinct nor convertible. Third, we push the utility constraints into Apriori and use them to prune candidate itemsets. Neither of the above two approaches addressed this.

Finally, our work is different from existing research on "interestingness" [8, 13], which focuses on finding "interesting patterns" by matching them against a given set of user's beliefs. Informally, a derived association rule is considered "interesting" if it conforms to or conflicts with the user's beliefs. In contrast, in OOA mining we measure the interestingness of OOA rules in terms of their probabilities as well as their utilities in supporting the user's objective.

\section{Objective, Support, Confidence, and Utility}

We assume that readers are familiar with traditional association rule mining, especially with the widely used Apriori algorithm [1]. A data base or dataset $D B$ is associated with a finite set $D B_{a t t}$ of attributes. Each attribute $A_{i}$ has a finite domain $V_{i}$ (continuous attributes can be discretized using methods such as that in [3]). For each $v \in V_{i}, A_{i}=v$ is called an item. An itemset or a pattern is a set of items. A $k$-itemset is an itemset with $k$ items. $D B$ consists of a finite set of records/transactions built from $D B_{\text {att }}$, with each record being a set $\left\{A_{1}=v_{1}, \ldots, A_{m}=v_{m}\right\}$ of items where $A_{i} \neq A_{j}$ for any $i \neq j$. We use $|D B|$ to denote the total number of records in $D B$. Finally, for any itemset $I$ the function $\operatorname{count}(I, D B)$ returns the number of records in $D B$ that are supersets of $I$.

An objective describes anything that we want to achieve or we are interested in. In order to discover patterns in a dataset $D B$ that support our objective $O b j$, we need first to formulate $O b j$ in terms of items of $D B$. This can be done by first partitioning $D B_{a t t}$ into two disjoint subsets: $D B_{a t t}=D B_{a t t}^{O b j} \cup D B_{a t t}^{n O b j}$ where each attribute $A \in$ $D B_{a t t}^{O b j}$ obviously contributes to $O b j$, whereas each $A \in$ $D B_{a t t}^{n O b j}$ does not. For convenience, we refer to attributes in $D B_{a t t}^{O b j}$ as objective attributes.

Let $A$ be an objective attribute and $V$ its domain. For each $v \in V, A=v$ is called an objective item or a class of $A$. We use $\operatorname{class}(A)$ to denote all classes of $A$. Let $\Re$ be a relation symbol such as $=,>$, <, etc. For each $v \in V$, $A \Re v$ is called an objective relation. An objective can then be represented by a logic formula over objective relations using the connectives $\wedge, \vee$ or $\neg$. Formally, we have

Definition 1 An objective $O b j$ over a dataset $D B$ is a disjunctive normal form $C_{1} \vee \ldots \vee C_{m}(m \geq 1)$ where each $C_{i}$ is a conjunction $D_{1} \wedge \ldots \wedge D_{n}(n \geq 1)$ with each $D_{j}$ being an objective relation or the negation of an objective relation.

With an objective $O b j$ as formulated above, we can then evaluate against a dataset how a pattern $I=\left\{I_{1}, \ldots, I_{m}\right\}$ statistically and semantically supports $O b j$ by defining the support, confidence and utility of the corresponding rule $I_{1}, \ldots, I_{m} \rightarrow O b j$. In OOA mining, we say an objective Obj holds in a record $r$ in $D B$ (or we say $r$ supports $O b j$ ) if $O b j$ is true given $r$. Furthermore, for any itemset $I=$ $\left\{I_{1}, \ldots, I_{m}\right\}$ we say $I \cup\{O b j\}=\left\{I_{1}, \ldots, I_{m}, O b j\right\}$ holds in $r$ if both $O b j$ and all $I_{i}$ s are true in $r$. We then extend the function $\operatorname{count}(I, D B)$ to $\operatorname{count}(I \cup\{O b j\}, D B)$ that returns the number of records in $D B$ in which $I \cup\{O b j\}$ holds.

Definition 2 Let $I_{1}, \ldots, I_{m} \rightarrow \operatorname{Obj}(s \%, c \%, u)$ be an association rule in OOA mining. Then the support and confidence of the rule are respectively given by

$$
\begin{aligned}
& s \%=\frac{\operatorname{count}\left(\left\{I_{1}, \ldots, I_{m}, O b j\right\}, D B\right)}{|D B|} * 100 \%, \\
& c \%=\frac{\operatorname{count}\left(\left\{I_{1}, \ldots, I_{m}, O b j\right\}, D B\right)}{\operatorname{count}\left(\left\{I_{1}, \ldots, I_{m}\right\}, D B\right)} * 100 \% .
\end{aligned}
$$

Let $O b j$ be an objective and $A$ an objective attribute. Based on $O b j$, the classes of $A$ can be subjectively classified into three disjoint groups: $\operatorname{class}(A)=\operatorname{class}^{+}(A) \cup$ class $^{-}(A) \cup$ class $^{\circ}(A)$ where class $^{+}(A)$ consists of all classes of $A$ that show positive support for $\mathrm{Obj} \mathrm{class}^{-}(A)$ of all classes of $A$ that show negative support for $O b j$, and $\operatorname{class}^{\circ}(A)$ of all classes of $A$ that show neither positive nor negative support for $O b j$. Therefore, classes in class $^{+}(A)$ will bring $O b j$ positive utilities, whereas classes in class $^{-}(A)$ bring negative utilities. We then associate each class $A=v$ in $\operatorname{class}^{+}(A)$ or $\operatorname{class}^{-}(A)$ with a utility 
$u_{A=v}$ (a real number). Since any class in $\operatorname{class}^{\circ}(A)$ can be considered as a special positive class with a utility 0 , we can merge $\operatorname{class}^{\circ}(A)$ into the positive group. Therefore, in the sequel we always assume that any class $A=v$ belongs to either class $^{+}(A)$ or class $^{-}(A)$. The groups of positively and negatively supporting classes of a dataset $D B$ for $O b j$ are then respectively defined as follows: $\operatorname{class}^{+}(D B)=$ $\left\{A=v\left(u_{A=v}\right) \mid A \in D B_{\text {att }}^{O b j}\right.$ and $\left.A=v \in \operatorname{class}^{+}(A)\right\}$ and class $^{-}(D B)=\left\{A=v\left(u_{A=v}\right) \mid A \in D B_{a t t}^{O b j}\right.$ and $A=v \in$ class $\left.^{-}(A)\right\}$.

An OOA itemset (or OOA pattern) is a set $\left\{A_{1}=\right.$ $\left.v_{1}, \ldots, A_{m}=v_{m}\right\}$ of items with $A_{i} \in D B_{a t t}^{n O b j}$ and $A_{i} \neq A_{j}$ for any $i \neq j$. Let $I$ be an OOA itemset and $r$ a record in $D B$ with $I \subseteq r$. Let $C_{r}$ be the set of classes in $r$. The positive utility $u_{r}^{+}(I)$ (resp. negative utility $u_{r}^{-}(I)$ ) of $r$ for $I$ is the sum of the utilities of all positively (resp. negatively) supporting classes in $C_{r}$, given by

$$
\begin{aligned}
& u_{r}^{+}(I)=\sum_{A=v \in C_{r} \wedge A=v\left(u_{A=v}\right) \in c^{\prime a s s}{ }^{+}(D B)} u_{A=v}, \\
& u_{r}^{-}(I)=\sum_{A=v \in C_{r} \wedge A=v\left(u_{A=v}\right) \in \text { class }^{-}(D B)} u_{A=v},
\end{aligned}
$$

The positive and negative utility of $D B$ for $I$ are then

$$
\begin{aligned}
u_{D B}^{+}(I) & =\sum_{r \in D B \wedge I \subseteq r} u_{r}^{+}(I), \\
u_{D B}^{-}(I) & =\sum_{r \in D B \wedge I \subseteq r} u_{r}^{-}(I) .
\end{aligned}
$$

Definition 3 Let $I_{1}, \ldots, I_{m} \rightarrow O b j(s \%, c \%, u)$ be an association rule with $I=\left\{I_{1}, \ldots, I_{m}\right\}$ an OOA itemset. Let $u_{D B}(I)=u_{D B}^{+}(I)-u_{D B}^{-}(I)$. The utility of the rule (or the itemset $I$ ) is given by

$$
u=\frac{u_{D B}(I)}{\operatorname{count}(I, D B)}
$$

Example 1 Let us consider a simplified dataset $D B_{1}$ about medical treatments for a certain disease as shown in Table 1, where treatment, effectiveness and side-effect are attributes with domains $\{1,2, \ldots, 5\},\{1,2, \ldots, 5\}$ and $\{1,2,3,4\}$, respectively. $R \#$ is not an attribute of $D B_{1}$. It is used to identify records by assigning a unique number to each record. Table 2 shows the degrees of the effectiveness and side-effects which are assigned by experienced domain experts. The doctor then wants to discover from $D B_{1}$ the best treatments with high effectiveness and low side-effects. Apparently, this is a typical objective-oriented utility-based mining problem.

The objective $O b j$ is "high effectiveness with low side-effects," which divides the set of attributes $D B_{1_{\text {att }}}$ into $D B_{1_{\text {att }}}^{O b j}=\{$ effectiveness, side-effect $\}$ and
Table 1. A medical dataset $D B_{1}$.

\begin{tabular}{|c|c|c|c|}
\hline$R \#$ & treatment & effectiveness & side-effect \\
\hline 1 & 1 & 2 & 4 \\
2 & 2 & 4 & 2 \\
3 & 2 & 4 & 2 \\
4 & 2 & 2 & 3 \\
5 & 2 & 1 & 3 \\
6 & 3 & 4 & 2 \\
7 & 3 & 4 & 2 \\
8 & 3 & 1 & 4 \\
9 & 4 & 5 & 2 \\
10 & 4 & 4 & 2 \\
11 & 4 & 4 & 2 \\
12 & 4 & 3 & 1 \\
13 & 5 & 4 & 1 \\
14 & 5 & 4 & 1 \\
15 & 5 & 4 & 1 \\
16 & 5 & 3 & 1 \\
\hline
\end{tabular}

Table 2. Degrees of the effectiveness and side-effects.

\begin{tabular}{|ll|ll|}
\hline & effectiveness & & \multicolumn{1}{c|}{ side-effect } \\
\hline 5 & getting much better & 4 & very serious \\
4 & getting better & 3 & serious yet tolerable \\
3 & no obvious effect & 2 & a little \\
2 & getting worse & 1 & normal \\
1 & getting much worse & & \\
\hline
\end{tabular}

$D B_{1_{\text {att }}}^{n O b j}=\{$ treatment $\}$. Based on the measurement of the effectiveness and side-effects (Table 2), Obj may be formulated by the formula: (effectiveness $>3$ ) $\wedge$ (sideeffect $<3$ ). Assume we are given the following groups of positively and negatively supporting classes (eff stands for effectiveness and sid for side-effect):

$$
\begin{gathered}
\text { class }^{+}\left(D B_{1}\right)=\{\text { eff }=5(1), \text { ef } f=4(0.8), \\
\text { ef } f=3(0), \text { sid }=1(0.6), \text { sid }=2(0)\}, \\
\text { class }^{-}\left(D B_{1}\right)=\{\text { eff } f=1(1), \text { ef } f=2(0.8), \\
\text { sid }=4(0.8), \text { sid }=3(0.4)\} .
\end{gathered}
$$

Table 3 shows the supports, confidences and utilities for all rules of the form "treatment $=k \rightarrow O b j$ " where $k$ is a treatment number, which are composed from the dataset $D B_{1}$. Note that the last two rules have quite different utilities for the objective, although their support and confidence are the same. Therefore, "treatment $=5$ " should be the best because it has the highest utility in supporting the objective. 
Table 3. Supports, confidences and utilities.

\begin{tabular}{|l|l|l|l|}
\hline \multicolumn{4}{|c|}{ Obj $:($ effectiveness $>3) \wedge($ side-effect $<3)$} \\
\hline rules & $s \%$ & $c \%$ & $u$ \\
\hline treatment $=1 \rightarrow O b j$ & 0 & 0 & -1.6 \\
treatment $=2 \rightarrow O b j$ & $12.5 \%$ & $50 \%$ & -1 \\
treatment $=3 \rightarrow O b j$ & $12.5 \%$ & $66 \%$ & -0.2 \\
treatment $=4 \rightarrow O b j$ & $18.75 \%$ & $75 \%$ & 0.8 \\
treatment $=5 \rightarrow O b j$ & $18.75 \%$ & $75 \%$ & 1.2 \\
\hline
\end{tabular}

\section{Mining OOA Rules}

\subsection{Objective-Oriented Apriori}

Definition 4 Let $D B$ be a dataset and $O b j$ an objective. Let $m s \%, m c \%$ and $m u$ be a user-specified minimum support, minimum confidence and minimum utility, respectively. Let $I=\left\{I_{1}, \ldots, I_{m}\right\}$ be an OOA itemset. $I$ is an $O O A$ frequent pattern/itemset in $D B$ if $s \% \geq m s \%$. Let $I$ be an OOA frequent pattern. $I_{1}, \ldots, I_{m} \rightarrow O b j(s \%, c \%, u)$ is an $O O A$ association rule (OOA rule) if $c \% \geq m c \%$ and $u \geq m u$. Here $s \%, c \%$ and $u$ are as defined in Equations (3), (4) and (9), respectively.

OOA mining is then to derive all OOA rules from $D B$. We extend Apriori [1] to generating OOA frequent patterns and rules by enhancing it with mechanisms for handling objectives and utilities. For convenience, we refer to the extended algorithm as Objective-Oriented Apriori (OOApriori).

For the data structure, we associate each OOA itemset with some necessary data fields to record data like counts and utilities. This is done by organizing an itemset into a structure using pseudo $C^{++}$language. That is, each OOA itemset $I=\left\{I_{1}, \ldots, I_{m}\right\}$ is internally an instance of the data type ITEMSET defined as follows:

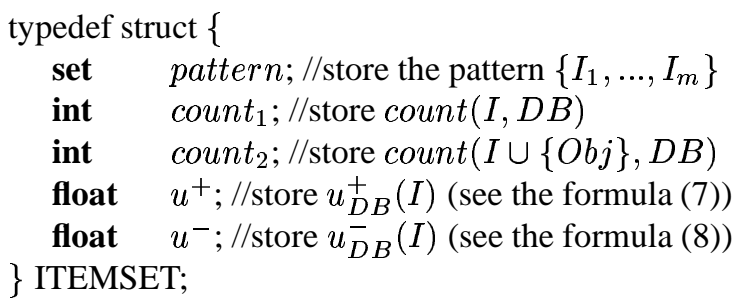

We use $I . D$ to refer to the field $D$ of $I$. I.count $t_{1}$, I.count ${ }_{2}, I . u^{+}$and $I . u^{-}$are all initialized to 0 when $I$ is created. Moreover, when no confusion would occur, by $I$ we refer to its pattern I.pattern $=\left\{I_{1}, \ldots, I_{m}\right\}$.

\section{Algorithm 1: Objective-Oriented Apriori.}

Input: $m s \%, m c \%, m u, O b j$ and $D B$.

Output: $F P$, the set of OOA frequent itemsets, and
$A R$, the set of OOA rules.

function $O O A$ priori $(m s \%, m c \%, m u, O b j, D B)$

1) $A R=F P=\emptyset$;

2) $k=1$;

3) $C_{k}=\{I \mid I$ is an OOA 1 -itemset in $D B\}$;

//Part 1: Collect counts and utilities of $k$-itemsets

4) for each record $r$ in $D B$

5) for each $k$-itemset $I \in C_{k}$

6) if $I \subseteq r$ then begin

7) I.count $1++$;

8) $\quad I . u^{+}=I \cdot u^{+}+u_{r}^{+}(I)$;

9) $\quad I . u^{-}=I \cdot u^{-}+u_{r}^{-}(I)$;

10) if $O b j$ holds in $r$ then

11) I.count ${ }_{2}++$

12) end

//Part 2: Check for frequent patterns $\left(L_{k}\right)$ and rules $(A R)$

13) $L_{k}=\emptyset$;

14) for each $I=\left\{I_{1}, \ldots, I_{k}\right\} \in C_{k}$

15) if $s \%=\frac{I . \text { count }_{2}}{D B} \geq m s \%$ then begin

16) $\quad L_{k}=L_{k} \cup\{I\}$

17) $\quad c \%=\frac{I . \text { count }_{2}}{I . \text { count }_{1}}$;

18) $\quad u=\frac{I \cdot u^{+}-I . u^{-}}{\text {I.count }}$;

19) if $c \% \geq m c \%$ and $u \geq m u$ then

20) $\quad A R=A R \cup\left\{I_{1}, \ldots, I_{k} \rightarrow \operatorname{Obj}(s \%, c \%, u)\right\}$

21) end

//Part 3: Generate $(k+1)$-itemsets

22) if $L_{k} \neq \emptyset$ then begin

23) $k++$;

24) $\quad C_{k}=\operatorname{aprioriGen}\left(L_{k-1}\right)$; //New candidate itemsets

25) goto 4)

26) end

27) return $F P=\bigcup_{i} L_{i}$ and $A R$

end

In Algorithm 1, for each $k \geq 1 C_{k}$ is used to store candidate frequent OOA $k$-itemsets, $L_{k}$ to store frequent OOA $k$-itemsets, and $A R$ to store all OOA rules. OOApriori consists of three major parts. The first part (lines 4-12) scans the dataset $D B$ and applies each record in $D B$ to counting the frequency and computing the positive and negative utilities of each candidate itemset in $C_{k}$. At lines 8 and 9, $u_{r}^{+}(I)$ and $u_{r}^{-}(I)$ are as defined in Equations (5) and (6). The second part (lines 13-21) checks the support, confidence and utility of each candidate itemset $I=\left\{I_{1}, \ldots, I_{k}\right\}$ in $C_{k}$ against the three user-specified minimums $m s \%$, $m c \%$ and $m u$ to see if $I$ is an OOA frequent pattern and $I_{1}, \ldots, I_{k} \rightarrow O b j$ is an OOA rule. After all OOA frequent $k$-itemsets and rules have been generated, the third part (lines 22-26) of OOApriori generates new candidate $(k+1)$-itemsets based on $L_{k}$ by calling the following function aprioriGen (). This function is borrowed from Apriori [1].

function aprioriGen $\left(L_{k}\right)$ 
1) $C_{k+1}=\emptyset$

2) for each pair of itemsets in $L_{k}$ of the form

3)

4) $\left\{I_{1}, \ldots, I_{k-1}, I_{k}\right\}$ and $\left\{I_{1}, \ldots, I_{k-1}, I_{k+1}\right\}$ $C_{k+1}=C_{k+1} \cup\left\{\left\{I_{1}, \ldots, I_{k+1}\right\}\right\}$;

//Prune itemsets

5) for each $I \in C_{k+1}$

6) if some $k$-sub-itemset of $I$ is not in $L_{k}$ then

7) $\quad C_{k+1}=C_{k+1}-\{I\}$;//Remove $I$ from $C_{k+1}$

8) return $C_{k+1}$

end

After the set $C_{k+1}$ of new candidate itemsets has been generated, the process goes to the next cycle (line 25) for deriving OOA frequent $(k+1)$-itemsets and rules. OOApriori will continue this way until no new OOA frequent itemsets can be generated (line 22).

Theorem 1 If $I=\left\{I_{1}, \ldots, I_{m}\right\}$ is an OOA frequent pattern and $J \subset I$ with $J \neq \emptyset$, then $J$ is an OOA frequent pattern.

Theorem 2 OOApriori is sound and complete in the sense that $I$ is an OOA frequent itemset if and only if $I \in F P$ and that $I_{1}, \ldots, I_{m} \rightarrow \operatorname{Obj}(s \%, c \%, u)$ is an OOA rule if and only if it is in $A R$.

\subsection{A Pruning Strategy for Mining OOA Rules}

Theorem 2 shows the correctness of applying OOApriori to computing OOA frequent itemsets and rules. In this section we develop a pruning strategy to improve its efficiency. Here and throughout, when we say that an OOA itemset $I=\left\{I_{1}, \ldots, I_{m}\right\}$ passes/violates the confidence or the utility constraint, we mean that the OOA rule $I_{1}, \ldots, I_{m} \rightarrow O b j$ passes/violates the constraint.

Four types of constraints for association mining have been identified in the literature $[10,11]$. Let $C$ be a constraint and $S_{1}$ and $S_{2}$ be two arbitrary itemsets. For $S_{1} \subset$ $S_{2}, C$ is anti-monotone if $S_{1}$ violating $C$ implies $S_{2}$ violates $C$, and $C$ is monotone if $S_{1}$ satisfying $C$ implies $S_{2}$ satisfies $C$. If $C$ is succinct then $S_{1}$ and $S_{2}$ satisfying $C$ implies $S_{1} \cup S_{2}$ satisfies $C$. $C$ is convertible if there exists an order $R$ on items such that for any itemset $S$ satisfying $C$, every prefix of $S$ w.r.t. $R$ satisfies $C$.

Theorem 1 assures us that the support constraint for OOA frequent patterns is anti-monotone. Therefore, in OOApriori we can safely delete an itemset $I$ from $L_{k}$ when its support is below the minimum support (see line 15) because no frequent patterns will be built from $I$. It turns out, however, that neither the confidence nor the utility constraint for OOA rules is anti-monotone.

Theorem 3 The utility constraint for OOA rules is neither monotone nor anti-monotone nor succinct nor convertible.
The pruning problem is then described as follows: For any itemset $I$ in $L_{k}$ (see the OOApriori algorithm) that has passed the support constraint but violates either the confidence or the utility constraint, can we delete $I$ from $L_{k}$ without missing any OOA rules? Without any pruning mechanism, OOApriori will generate all OOA frequent items, many of which may produce no OOA rules because of the violation of the confidence or the utility constraint. Look at the function aprioriGen $\left(L_{k}\right)$ again. Since all $(k+1)$-itemsets are composed from the $k$-itemsets in $L_{k}$, we need to keep $L_{k}$ as small as possible by removing some OOA frequent itemsets from which no OOA rules would be possibly built.

We present a pruning strategy using the support and utility constraints. To describe the pruning strategy, we add two more data fields to the internal structure of an OOA itemset $I$ as shown below:

typedef struct \{
set pattern; //store the pattern $\left\{I_{1}, \ldots, I_{m}\right\}$
int count $_{1} ; / /$ store $\operatorname{count}(I, D B)$
int count $_{2} ; / /$ store $\operatorname{count}(I \cup\{O b j\}, D B)$
float $u^{+} ; / /$store $u_{D B}^{+}(I)$
float $u^{-} ; / /$store $u_{D B}^{-}(I)$
int $\quad$ count $t_{2}^{+} ; / /$store $\left|S^{+}\right|$
float lnu;//store the least negative utility
ITEMSET;

Here, let $S$ be the set of records in $D B$ in which $I \cup\{O b j\}$ holds and $S^{+}$be the set of records in $S$ which contain no negative class (i.e., all classes of these records are in class $^{+}(D B)$ ), then the first new field count $_{2}^{+}$is used to store $\left|S^{+}\right|$(note that the field count $_{2}$ stores $|S|$ ) and the second new field $\ln u$ is used to store the least negative utility of a record in $S-S^{+}$, i.e. $\ln u \leq u_{r}^{-}(I)$ for any $r$ in $S-S^{+}$.

Strategy 1 Remove any OOA itemset $I=\left\{I_{1}, \ldots, I_{k}\right\}$ from $L_{k}$ if $I$.count $t_{2}^{+}<m s \% *|D B|$ and $\frac{I . u^{+}-L B^{-}}{m s \% * D B \mid}<m u$, where $L B^{-}=\left(m s \% *|D B|-I\right.$.count $\left.t_{2}^{+}\right) * I . \ln u$.

Since $I$ is a frequent OOA itemset, there are at least $m s \% *|D B|$ records in $|D B|$ in which $I \cup\{O b j\}$ holds. When I.count $_{2}^{+}<m s \% *|D B|$, there are at least $(m s \% *$ $|D B|-$ I.count $\left._{2}^{+}\right)$records in $D B$ in which $I \cup\{O b j\}$ holds that contain negative classes. Therefore, $L B^{-}>0$ is the least negative utility of $D B$ for $I$ and thus is the lower bound of $I . u^{-}$. As a result, $I . u^{+}-L B^{-}$is the upper bound of the utility of $D B$ for $I$. To sum up, this strategy says that an OOA frequent itemset $I$ is removable if the upper bound of its expected utility is below the minimum utility. The following theorem shows that applying this strategy will not miss any OOA rules. 
Theorem 4 Let $I=\left\{I_{1}, \ldots, I_{k}\right\}$ be an OOA frequent itemset. If I.count ${ }_{2}^{+}<m s \% *|D B|$ and $\frac{I . u^{+}-L B^{-}}{m s \% * D B \mid}<m u$ then there is no OOA itemset $J=\left\{J_{1}, \ldots, J_{n}\right\} \supseteq I$ such that $J_{1}, \ldots, J_{n} \rightarrow O b j$ is an OOA rule.

It is easy to push Strategy 1 into the OOApriori algorithm. This is done by replacing lines 14-21 of Algorithm 1 with the following lines:

14) for each $I=\left\{I_{1}, \ldots, I_{k}\right\} \in C_{k}$

15) if $s \%=\frac{I \text {.count } 2}{D B} \geq m s \%$ then begin

16) $L_{k}=L_{k} \cup\{I\}$;

17) $\quad c \%=\frac{I . \text { count }_{2}}{\text { I.count }}$;

18) $u=\frac{I \cdot u^{+}-I . u^{-}}{\text {I.count } 1}$;

19) if $c \% \geq m c \%$ and $u \geq m u$ then

20) $\quad A R=A R \cup\left\{I_{1}, \ldots, I_{k} \rightarrow \operatorname{Obj}(s \%, c \%, u)\right\}$;

20-1) else begin

20-2) $\quad L B^{-}=\left(m s \% *|D B|-\right.$ I.count $\left.t_{2}^{+}\right) *$ I.lnu;

20-3) if $I$. count ${ }_{2}^{+}<m s \% *|D B|$ and $\frac{I . u^{+}-L B^{-}}{m s \% * D B}$

20-4) $\quad<m u$ then $L_{k}=L_{k}-\{I\} / /$ by Strategy 1

20-5) end

21) end

The above procedure works as follows: For each candidate $k$-itemset in $C_{k}$, if it passes the support constraint then it is added to $L_{k}$ (lines 15 and 16). If it also passes both the confidence and the utility constraint, an OOA rule built from $I$ is added to $A R$ (lines 17-20). Otherwise, when $I$ passes the support constraint but violates either the confidence or the utility constraint, our pruning strategy is applied (lines 20-1 to 20-5) to remove some OOA frequent itemsets from $L_{k}$ from which no OOA rules will be produced. The correctness of the OOApriori algorithm enhanced with the pruning strategy follows immediately from Theorems 2 and 4 . That is, $I_{1}, \ldots, I_{m} \rightarrow \operatorname{Obj}(s \%, c \%, u)$ is an OOA rule if and only if it is in $A R$.

\section{Experimental Evaluation}

We show the effect of applying our pruning strategy by empirical experiments. We choose the widely used German Credit dataset from the UCI Machine Learning Archive (ftp://ftp.ics.uci.edu/pub /machine-learningdatabases/statlog/german/). This dataset consists of 1000 records (each record represents a customer) with 21 attributes such as Status, Duration, Credit-history, Purpose, Employment, etc. The last attribute Conclusion classifies a customer as good or bad in terms of his/her credits. The reason we use this dataset in our experiment is that its attributes are semantically easy to understand so that we can flexibly create different objectives from them to test our approach.

We build four datasets with different sizes from the 1000 records. $D S_{1}$ consists of 600 records, $D S_{2}$ of 700 records, $\ldots$, and $D S_{4}$ of 900 records. The objective attributes are Liable-people, Foreign and Conclusion, and the objective $\mathrm{Obj}$ is defined as (Conclusion=good) $\wedge$ (Liable-people $=2$ $\vee$ Foreign=no). That is, suppose we are interested in customers whose credit is good and who either are not foreign workers or have more than one person being liable to provide maintenance for the credit account. All the remaining eighteen attributes are treated as non-objective attributes. The utilities of the major classes of the objective are defined in Table 4 where we normalize the utilities into $[0,100]$.

Table 4. Class utilities.

\begin{tabular}{|c|c|c|c|}
\hline & Conclusion & Foreign & Liable-people \\
\hline class $^{+}(D B)$ & good (70) & no (10) & $2(20)$ \\
\hline class $^{-}(D B)$ & bad (70) & yes $(10)$ & $1(20)$ \\
\hline
\end{tabular}

Let $N_{1}$ and $N_{2}$ be the sizes of the two sets of OOA candidate itemsets generated by OOApriori with and without applying Strategy 1, respectively. We evaluate the effect of applying Strategy 1 to pruning OOA itemsets by demonstrating its itemset reduction rate defined by $\frac{N_{2}-N_{1}}{N_{2}}$. Figure 1 shows our experimental results on the itemset reduction rates where we use different minimum utilities while keeping the minimum support and minimum confidence unchanged. The results strongly demonstrate that applying our pruning strategy can greatly improve the efficiency of the OOApriori algorithm. On average, they pruned $8 \%-9 \%$ of the candidate itemsets during the mining process. Figure 2 further demonstrates the effectiveness of the pruning strategy, where we use the same minimum confidence and minimum utility while letting the minimum support vary.

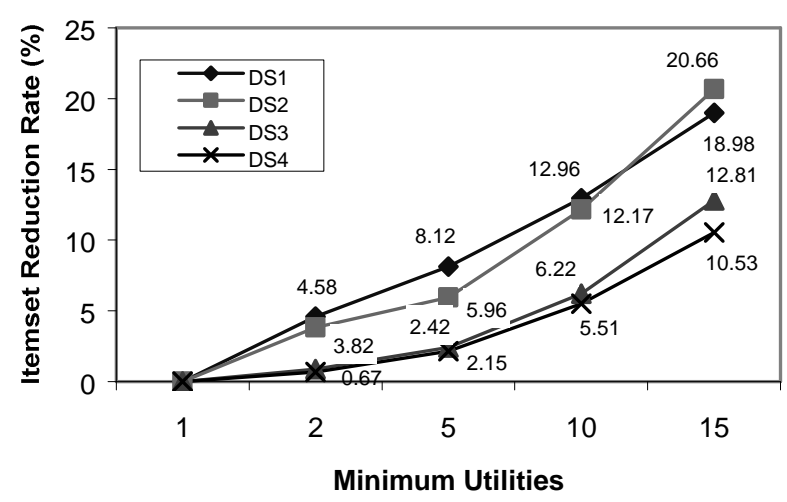

\section{Figure 1. The itemset reduction rates against minimum utilities.}

\section{Conclusions}

We have developed a new approach to modeling association patterns. OOA mining discovers patterns that are 


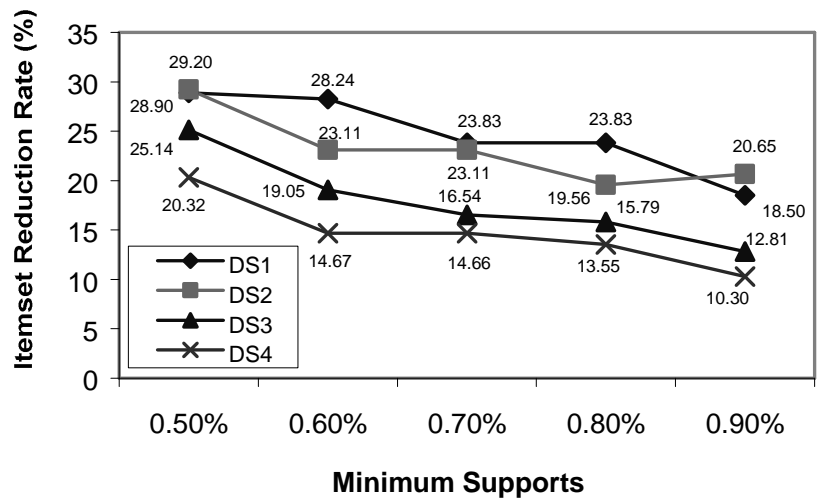

Figure 2. The itemset reduction rates against minimum supports.

explicitly relating to a given objective that a user wants to achieve or is interested in and its utility. As a result, all OOA rules derived from a dataset by OOA mining are useful because applying them would increase business utility of an enterprise. This shows a significant difference from traditional association mining.

We developed an algorithm for mining OOA frequent patterns and rules. The algorithm is an enhancement to Apriori with specific mechanisms for handling objective utility. Since the utility constraint is neither anti-monotone nor monotone nor succinct nor convertible, finding effective pruning strategies is of great significance. We developed a novel pruning strategy for mining OOA rules by combining the support and utility constraints. As far as we can determine, no similar work has been reported in the literature.

\section{Acknowledgement}

Yi-Dong Shen is supported in part by Chinese National Natural Science Foundation, Trans-Century Training Program Foundation for the Talents by the Chinese Ministry of Education, and Foundations from Chinese Academy of Sciences. Qiang Yang thanks NSERC and IRIS-III program for their support.

\section{References}

[1] R. Agrawal and R. Srikant. Fast algorithm for mining association rules. In $V L D B$, pages 487-499, 1994.

[2] R. Bayardo, R. Agrawal, and D. Gounopolos. Constraint-based rule mining in large, dense databases. In ICDE, pages 188-197, 1999.
[3] J. Dougherty, R. Kohavi and M. Sahami. Supervised and unsupervised discretization of continuous features. ICML, 1995.

[4] J. Han, J. Pei, and Y. Yin. Mining frequent patterns without candidate generation. In SIGMOD, pages 1$12,2000$.

[5] R. Howard. Risk preference. In R. Howard and J. Matheson, eds. Readings in Decision Analysis, pages 429-465, 1977.

[6] J. Kleinberg, C. Papadimitriou, and P. Raghavan. A microeconomic view of data mining. Journal of Data Mining and Knowledge Discovery, 6(1):83-105, 1998.

[7] T. Lin, Y. Yao, and E. Louie. Value added association rules. In PAKDD, pages 328-333, 2002.

[8] B. Liu, W. Hsu, S. Chen, and Y. Ma. Analyzing the subjective interestingness of association rules. IEEE Intellgent Systems, 15:47-55, 2000.

[9] B. Masand and G. Piatetsky-Shapiro. A comparison of approaches for maximizing business payoff of prediction models. In KDD, pages 195-201, 1996.

[10] R. Ng, L. Lakshmanan, J. Han, and A. Pang. Exploratory mining and pruning optimizations of constrained association rules. In SIGMOD, pages 13-24, 1998.

[11] J. Pei and J. Han. Constrained frequent pattern mining: a pattern-growth view. ACM SIGKDD Explorations (Special Issue on Constrained Data Mining) 2(2), 2002.

[12] S. Russell and P. Norvig. Artificial Intelligence: A Modern Approach. Englewood Cliffs, NJ: Prentice Hall, 1994.

[13] A. Silberschatz and A. Tuzhilin. What makes patterns interesting in knowledge discovery system. IEEE Trans. on Knowledge and Data Engineering, 8:970974, 1996.

[14] R. Srikant, Q. Vu, and R. Agrawal. Mining association rules with item constraints. In $K D D$, pages $67-73$, 1997.

[15] K. Wang, S. Zhou and J. Han. Profit mining: from patterns to actions. In EDBT, pages 70-87, 2002.

[16] J. Wijsen and R. Meersman. On the complexity of mining quantitative association rules. Journal of Data Mining and Knowledge Discovery, 2(3):263281, 1998. 